\title{
La presencia de los países árabes en Internet: un análisis a través de sus indicadores tecnológicos y de sus universidades
}

The presence of Arab countries in the Internet: an analysis through their technological indicators and its universities

\author{
Cristina Faba-PÉRez (1), Benjamín VARgas-Quesada (2), Khaldoon Mohammad Oglah AL-DwaiR (3), \\ Félix MOYA-ANEGóN (4) \\ (1) Departamento de Información y Comunicación, Universidad de Extremadura. Plaza Ibn Marwan, La Alcazaba, 06071 Badajoz \\ España. E-mail: cfabper@unex.es; (2) Departamento de Biblioteconomía y Documentación, Universidad de Granada. Campus \\ Cartuja, Colegio Máximo, 18071 Granada, España, E-mail: benjamin@ugr.es; (3) Universidad Aplicada Al-Balqa, Al-Salt 19117, \\ Jordania, khadoc@ugr.es; (4) Instituto de Políticas y Bienes Públicos, Consejo Superior de Investigaciones Científicas. C/Albasanz, \\ 26-28, 28037, Madrid, España, felix.demoya@cchs.csic.es.
}

\begin{abstract}
Resumen
Apenas existen investigaciones sobre los países árabes en Internet, por lo que la presencia del mundo árabe en la Web, en general, y de sus universidades, en particular, aún está por descubrir. Por ello, el objetivo del presente trabajo es analizar la presencia de dichos países en Internet aplicando dos tipos de indicadores: tecnológicos, que reflejen la situación detallada de cada país y la evolución general sufrida por el conjunto de los Estados Árabes; y webmétricos de análisis de características, que muestren la presencia y calidad de las universidades del mundo árabe en Internet. En concreto, se analizan los países árabes y los sitios web de las universidades de Oriente Medio y Norte de África (Zona MENA). Los resultados obtenidos y las conclusiones derivadas al respecto demuestran que, si bien el factor económico de los países influye en la presencia de los mismos en la Red y en la calidad de sus universidades, no debe analizarse de forma aislada puesto que otros factores como la población y la apertura sociopolítica o cultural de los países también repercuten en su presencia en Internet. Así mismo, en los últimos años, se ha observado una evolución general positiva de los Estados Árabes con respecto a su tecnología.
\end{abstract}

Palabras clave: Indicadores tecnológicos. Indicadores webmétricos. Presencia en Internet. Universidades árabes. Países árabes.

\section{Introducción}

Actualmente, cualquier persona, institución o país que pretenda ser visible para la comunidad nacional o internacional necesita estar presente en Internet. Los países árabes no están exentos de dicha premisa. Por eso, en este trabajo se analiza la presencia de los países árabes en la Red, en primer lugar, teniendo en cuenta diversos indicadores tecnológicos que reflejan la evolución de cada país con respecto a su tecnología, y en segundo lugar, analizando la presen-

\begin{abstract}
Little research has been carried out on the presence of the Arab countries on the Internet, which goes to prove that the presence of the Arab world in the Web in general and of these countries' universities, in particular, is still at an emergent stage. The purpose of this paper is to analyse the presence of the aforementioned countries on the Internet in 2009 by the application of two types of indicators: technological indicators, which reveal the development of each country and the general evolution of Arab States; and webometric indicators, which allow to ascertain the presence and quality of the universities of the Arab world on the Internet. In particular, the countries under analysis herein are those in the Middle East and North Africa (i.e. the MENA region) and the websites of their universities. The findings and conclusions of this research project reveal that, although the financial situation of the countries is an influential factor in explaining their presence on the Web and the quality of their universities, this element must not be considered isolated, as other factors, such as population and the social, political or cultural opening of these countries, also have an impact on them. Furthermore, in the last years there has been an overall positive evolution of Arab States in respect of their technology.
\end{abstract}

Keywords: Technological indicators. Webometrics indicators. Internet presence. Arab universities. Arab countries.

cia y calidad de sus universidades en la Red mediante el uso de indicadores webmétricos.

En relación a los indicadores tecnológicos referentes a las Tecnologías de la Información y de la Comunicación (TIC) de los países árabes, el Informe de la Cumbre Mundial sobre la Sociedad de la Información (citado por Al-Dwairi y Herrero-Solana, 2007) señala la diferencia entre TIC antiguas (receptores de radio y televisión, así como los teléfonos fijos) y TIC nuevas (teléfonos móviles, ordenadores personales y cone- 
xiones a Internet). Estas últimas, presentan mayor dependencia de la disponibilidad de energía o de instalaciones de recarga $y$, por tanto, son un bien consumible no disponible en todos los países pobres. Además, juegan un papel fundamental en los ámbitos social, económico y cultural generando una nueva era en la confrontación de la civilización (Al-Mobarek, 2008).

El mundo actual se caracteriza por el cambio rápido, tanto cuantitativo como cualitativo, a través de una creciente dependencia de las TIC, que ha afectado a todos los aspectos de la vida personal y profesional. Las TIC pueden contribuir a reducir la pobreza, mejorar la educación y la prestación de servicios de salud, y hacer más accesibles y responsables a los gobiernos con respecto a los ciudadanos. En este marco, el acceso a un ordenador personal es importante no sólo porque se trata de un dispositivo de información por sí mismo, sino también porque es el instrumento necesario para acceder a Internet, con todo lo que esto conlleva (Alawi, 2006).

Sin embargo, mientras que Internet se ha ido desarrollando en el mundo occidental durante los últimos años, su presencia como parte de la vida cotidiana en el mundo árabe es relativamente nueva. Las primeras conexiones a Internet en estos países datan de principios de los años 90 (por ejemplo, Túnez fue el primer país árabe en tener conexión a Internet en 1991) (AlDwairi y Herrero-Solana, 2007).

Con respecto a la presencia de las universidades árabes en la Red mediante el uso de indicadores webmétricos, el estudio de los mismos se enmarca en la Webometrics (Almind e Ingwersen, 1997), que consiste en un análisis cuantitativo de los fenómenos de la web, incluyendo, fundamentalmente, el análisis de enlaces y el análisis descriptivo o de características (Faba-Pérez, Guerrero-Bote y Moya-Anegón, 2005). Se han realizado numerosas investigaciones en el entorno universitario de diferentes países y zonas geográficas, usando ambos tipos de análisis (Tang y Thelwall, 2002; Smith y Thelwall, 2002; Wilkinson et al., 2003; Tang y Thelwall, 2003; Payne y Thelwall, 2004; Nwagwu y Agarin, 2008; Ortega y Aguillo, 2008ab; Payne y Thelwall, 2008ab; Chung et al., 2009; Arakaki y Willet, 2009; Asadi y Shekofteh, 2009; Caballero-Cortés, Faba-Pérez y Moya-Anegón, 2009; Ortega y Aguillo, 2009b; Moscovkin, 2010; Abrizah, Noorhidawati y Kiran, 2010; World Universities Ranking on the Web).

Sin embargo, en el caso concreto de los países árabes de la zona MENA, hay pocos estudios de la webmetría aplicada a las universidades de dicha zona (Noruzi, 2006; Farrag, 2006; Elgohary, 2008; Ortega y Aguillo, 2009a; AlDwairi, Faba-Pérez y Vargas-Quesada, 2010; Vargas-Quesada, Al-Dwairi, Faba-Pérez y Moya-Anegón, 2013).

Según Farrag (2006) y Elgohary (2008), en relación a las investigaciones webmétricas en los países árabes, cabría señalar una serie de características que marcan su situación actual: a) la webmetría como campo de investigación novedoso en el contexto del mundo árabe; b) la ausencia de grupos de investigación bien delimitados y financiados, tanto a nivel público como privado; c) el fomento de la investigación en el ámbito de la webmetría por parte de autores concretos, de forma individual, lo que no favorece ni el desarrollo de la investigación en esta disciplina, ni el asentamiento de su base teórica como campo de investigación emergente; d) la escasa relevancia que se le concede a la actualización y renovación de los sitios web de los países árabes en todos los ámbitos (tanto privados, como públicos), lo que supone una evidente traba para el fomento y desarrollo de la investigación en webmetría, a pesar de que se cuenta con asesores externos que están contribuyendo al fomento de la actualización de los sitios web académicos de países como Arabia Saudí y Jordania.

Con la finalidad de contribuir al desarrollo de los países árabes en la Red, el objetivo del presente trabajo es analizar la presencia de los mismos en Internet aplicando dos tipos de indicadores: indicadores tecnológicos, que reflejen la situación de cada país con respecto a su tecnología; e indicadores webmétricos de análisis de características, que permitan descubrir la presencia y calidad de sus universidades en Internet. La hipótesis de partida es que aquellos países con mayor desarrollo tecnológico y renta per cápita, también tendrán una presencia más elevada en la Red y de mejor calidad, posicionando sus universidades en los primeros puestos en el ranking webmétrico obtenido con el análisis de sus características web.

\section{Datos}

En la presente investigación se estudian los países árabes de la zona geográfica MENA (Middle East and North Africa), es decir, los países pertenecientes a la Liga Árabe (League of Arab States), y los sitios web de sus universidades. La Liga de Estados Árabes es un organismo internacional con Sede en Egipto que está formada por veintidós países que comparten muchas características, tales como la cultura, la religión, el idioma árabe y la historia (tabla I). 


\begin{tabular}{llll}
\hline País & Dominio & País & Dominio \\
\hline Arabia Saudí & SA & Libia & LY \\
\hline Argelia & DZ & Marruecos & MA \\
\hline Bahrein & BH & Mauritania & MR \\
\hline Comores & KM & Omán & OM \\
\hline Dijbuti & DJ & Palestina & PS \\
\hline Egipto & EG & Qatar & QA \\
\hline EAU & AE & Siria & SY \\
\hline Iraq & IQ & Somalia & SO \\
\hline Jordania & JO & Sudán & SD \\
\hline Kuwait & KW & Túnez & TN \\
\hline Líbano & LB & Yemen & YE \\
\hline
\end{tabular}

Tabla I. Países Árabes de la zona MENA y su código de dominio según la norma ISO 3166

Para recopilar los datos referentes a los indicadores tecnológicos 2009-2013 de los países en estudio, se ha utilizado la fuente de datos estadísticos International Telecommunication Union (ITU), organismo especializado de las Naciones Unidas para las Tecnologías de la Información y la Comunicación.

Para recopilar las universidades correspondientes a cada país y poder calcular los indicadores webmétricos, se han usado diferentes recursos: como fuente principal se ha utilizado Association of Arab Universities, Jordan, y como fuente internacional Universities Worldwide. Una vez obtenidos los diferentes nombres de las universidades, se ha procedido a buscar las URL de aquellas de las que se carecía. La información obtenida de las mismas ha dado como resultado un total de 203 universidades con sus correspondientes URL, de las que parte la investigación. Para completar dicho listado, mediante un comprobador de enlaces del mercado denominado Linkbot (versión 6.0), se ha examinado cada una de las URL extrayendo sus enlaces externos dirigidos hacia universidades de la zona no recogidas previamente, lo que ha aumentado el listado de universidades hasta 245 en 2007 y 268 en el año 2009 (población final de estudio webmétrico del presente trabajo). Un caso especial ha sido el de las Islas Comores, donde no se han encontrado las URL de sus universidades, por lo que no se han podido obtener datos al respecto.

\section{Metodología}

\subsection{Indicadores tecnológicos}

Los indicadores tecnológicos reflejan la evolución de los países árabes de la zona MENA con respecto a la tecnología, teniendo presente que no siempre todos los habitantes tienen acceso a los bienes de consumo que ésta proporciona. La mayoría de los indicadores analizados que se muestran a continuación para examinar detalladamente cada país árabe se han calculado con datos de 2009, si bien, en algún caso se ha trabajado con el año 2008. Algunos de ellos se han hallado en relación con la renta per cápita de cada uno de los países, mientras que otros se han calculado respecto al número de habitantes. Con respecto a la evolución sufrida por los Estados árabes en general, se han consultado datos desde 2010 hasta 2013 sobre líneas telefónicas, ordenadores, Internet e idioma.

\subsection{Indicadores webmétricos ponderados}

Una vez conocida la situación tecnológica de los países de la zona MENA, se analizan los sitios web de sus universidades en 2009 para comprobar la presencia y calidad de las mismas en la Red en dicho período. Para ello, se aplican 16 indicadores webmétricos de análisis de características ponderados que permiten obtener un ranking de los sitios web universitarios a través de las características que incluyen. Siguiendo la metodología de Al-Dwairi, Faba-Pérez y VargasQuesada (2010), los indicadores webmétricos o de características analizadas en cada web universitaria y su ponderación respectiva se describen a continuación. Es preciso señalar que el peso asignado a cada indicador depende de su importancia de cara a la recuperación de información en webs universitarias:

- Enlaces externos $(0,4)$ : úmero de enlaces dirigidos hacia sitios web diferentes al de procedencia.

- Densidad hipertextual $(0,2)$ : número de enlaces que desde un sitio web se hacen a documentos html.

- Densidad multimedia $(0,1)$ : número de imágenes y ficheros de audio y vídeo de cada sitio web.

- Páginas pequeñas $(0,2)$ : número de páginas de un sitio web cuyo peso es inferior a $3 \mathrm{~Kb}$.

- Páginas actualizadas $(0,3)$ : número de páginas de un sitio web que han sufrido cambios en el último mes desde que se creó, aprobó, se puso en circulación, se editó o se alteró.

- Metadatos $(0,3)$ : número de etiquetas meta de un sitio web. La importancia de estas etiquetas es que favorecen la localización y recuperación del sitio web por parte de los robots de los motores de búsqueda. 
- Metadatos Dublin Core $(0,3)$ : este indicador identifica los sitios web que contienen etiquetas meta, pero en concreto metadatos expresados en el vocabulario estándar Dublin Core (DC).

- Enlaces erróneos externos $(-0,4)$ / internos $(-0,4)$ : número de enlaces dirigidos hacia sitios web diferentes al de procedencia (externos) o dirigidos a otra página del propio sitio web (internos) que muestran algún tipo de error (fichero o servidor no encontrado, imposible establecer conexión, etc.).

- Anclajes erróneos $(-0,2)$ : número de enlaces hipertextual erróneos hacia partes de un mismo documento html.

- Páginas sin título $(-0,3)$ : número de páginas de los sitios web que no incluyen la etiqueta title en su cabecera o que ésta se encuentra vacía.

- Páginas sin atributos de imágenes $(-0,1)$ : número de las páginas de los sitio web que no disponen de atributos de imagen como alt, heigth, o width.

- Páginas antiguas $(-0,3)$ : número de páginas de los sitios web que no han sido actualizadas en los últimos seis meses previos al momento en que se realiza este estudio.

- Páginas lentas $(-0,2)$ : número de páginas que dentro del sitio web tardan de 20 a 30 segundos en cargarse.

- Páginas profundas $(-0,2)$ : número de páginas de un sitio web a las que solo se puede acceder tras pasar por un mínimo de cuatro páginas.

- Enlaces con problemas no críticos $(-0,2)$ : número de enlaces de los sitios web que presentan problemas temporales de mantenimiento.

La posición en el ranking de cada universidad viene determinada por el Peso final obtenido por cada una de ellas. Dicho Peso corresponde a la suma total de los valores obtenidos en cada indicador teniendo en cuenta su ponderación correspondiente.

\section{Resultados}

\subsection{TIC}

Con la finalidad de poder interpretar correctamente los resultados detallados correspondientes a los indicadores tecnológicos y webmétricos, es preciso conocer con antelación la distribución ordenada de los países de la zona ME-
NA por renta per cápita según datos del ITU 2008: renta alta (Qatar, Kuwait, Emiratos Árabes Unidos, Bahrein, Arabia Saudí y Omán); renta media (Libia, Líbano, Argelia, Túnez, Jordania, Marruecos, Iraq, Egipto y Siria; renta baja (Sudán, Palestina, Djibouti, Yemen, Mauritania y Comores). Con respecto a Somalia, aunque no se dispone de datos económicos en 2008 debido a su estado de caos social y económico, pertenece al grupo de los países con renta baja, según datos de años anteriores del ITU.

Del análisis de los indicadores tecnológicos de los países de la zona MENA descritos en la metodología se obtienen los resultados que se muestran a continuación. Para observar la evolución temporal sufrida por algunos de los indicadores estudiados en la presente investigación en cada país con respecto al año 2002 se puede consultar el trabajo de Al-Dwairi y HerreroSolana (2007).

\subsubsection{Líneas telefónicas}

Atendiendo al número de líneas telefónicas de cada uno de los países de la zona MENA por cada 100 habitantes en 2009, se obtiene que todos los países del Golfo con renta per cápita alta son los que cuentan con un mayor número de líneas telefónicas por habitante.

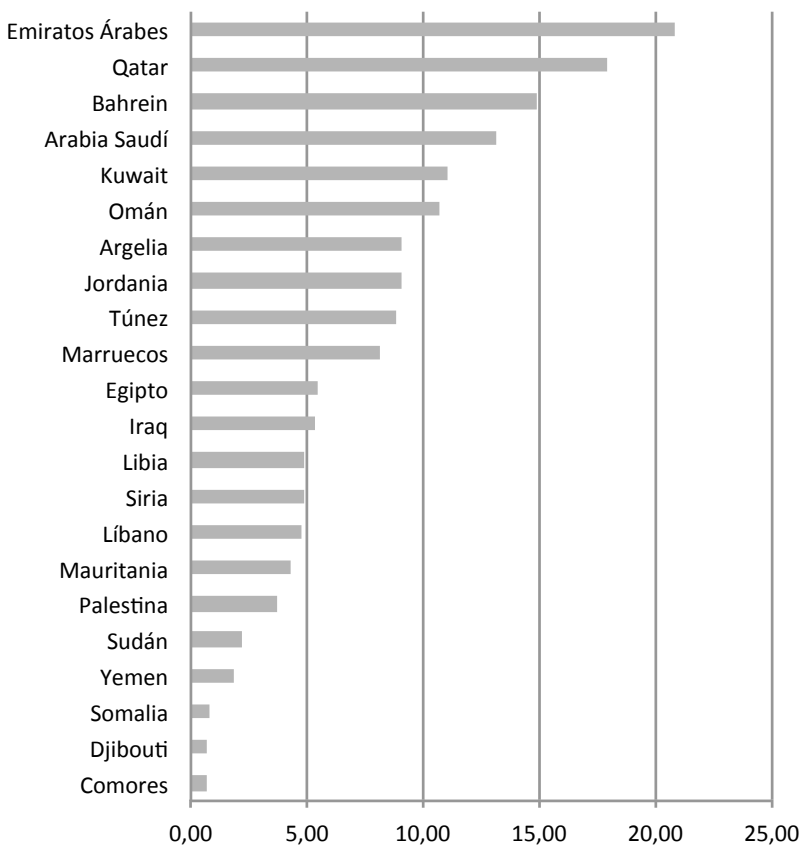

Figura 1. Líneas telefónicas por cada cien habitantes en 2009

A la cabeza se encuentran los Emiratos Árabes (con 20,81 líneas por cada 100 habitantes), seguido de Qatar $(17,86)$ y Bahrein $(14,92)$, aunque entre ellos existen grandes saltos cuantitati- 
vos. En la zona intermedia se hallan los países con renta per cápita media como Argelia $(9,05)$ y Líbano $(4,78)$ y, finalmente, en la última parte, se encuentran los países con menor renta per cápita como Djibouti $(0,69)$ o Comores $(0,68)$.

En la figura 2 se representan las líneas telefónicas por cada 100 habitantes en función de la renta per cápita. Hay tres grupos claramente diferenciados. En primer lugar, el grupo de los países cuya renta per cápita es mayor: en este caso, hay tres países que se sitúan por encima de la línea de tendencia (Emiratos Árabes (AE), Bahrein $(\mathrm{BH})$ y Arabia Saudí (SA)) sobresaliendo de forma especial los Emiratos Árabes con mayor número de líneas telefónicas por habitante respecto a su renta per cápita. En la parte intermedia se encuentra el grupo de los países con renta per cápita media, países que se sitúan casi en su totalidad por encima de la línea de tendencia, excepto Líbano (LB) y Libia (LY). El último grupo lo conforman los países con renta per cápita baja, donde se observa que todos, a excepción de Mauritania (MR) y Palestina (PS), se encuentran por debajo de la línea de tendencia, especialmente desfavorable son los casos de Comores (KM) y Djibouti (DJ). Somalia no aparece en la distribución por no disponer de datos económicos en el año 2008.

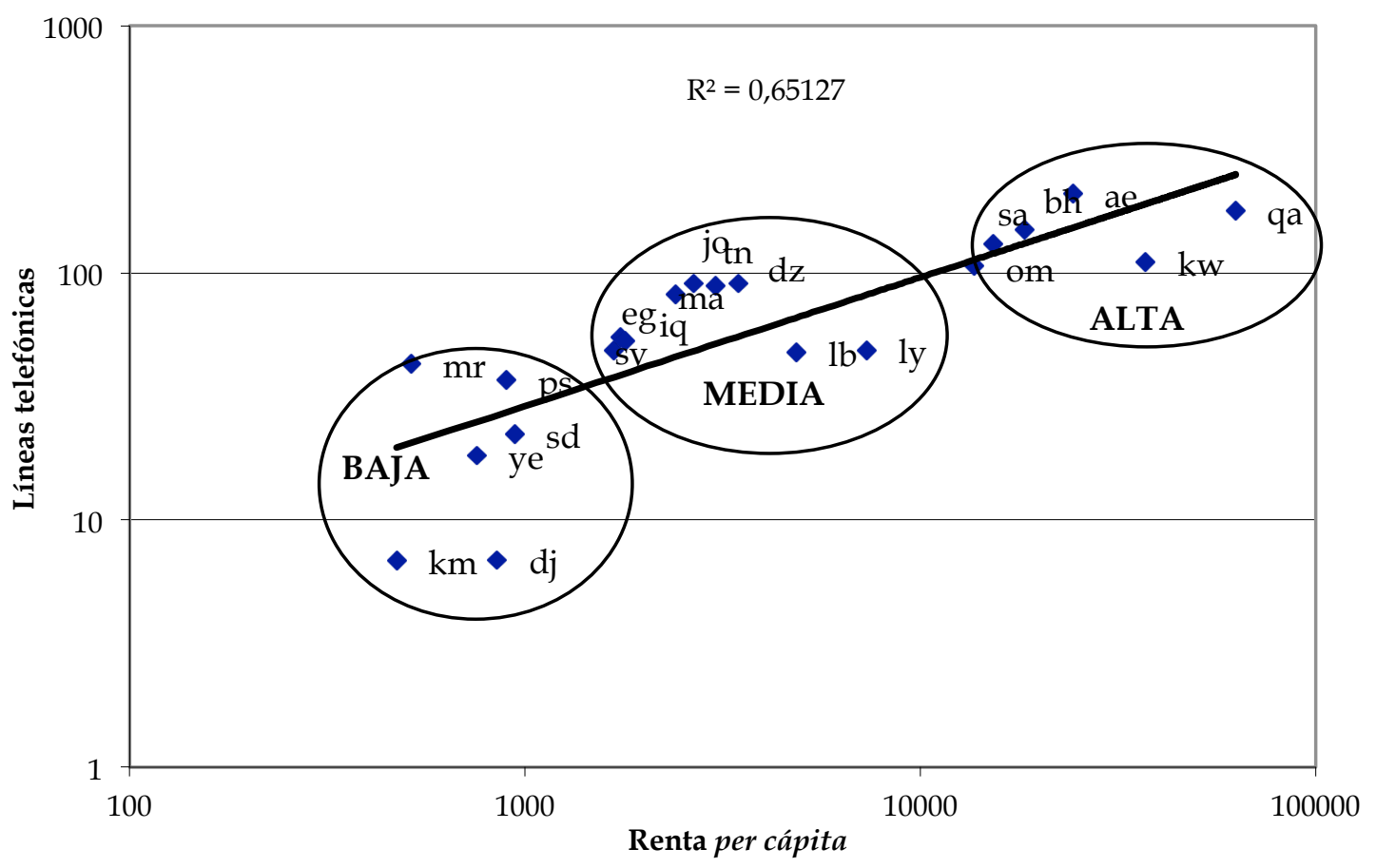

Figura 2. Líneas telefónicas totales en 2009 en función de la renta per cápita en el año 2008

Con respecto la evolución sufrida por los Estados Árabes desde 2010 hasta 2013 con respecto al número de líneas telefónicas fijas por cada 100 habitantes es el siguiente: $2010(9,8), 2011$ $(9,8), 2012(9,6), 2013(8,9)$, lo que supone un decrecimiento de 2013 con respecto a 2010 del $-9,57 \%$. Sin embargo, en caso de la telefonía móvil, las líneas se incrementan en un $23,82 \%$ : $2010(87,9), 2011(99,1), 2012(105,4)$ y 2013 $(108,8)$.

\subsubsection{Ordenadores}

El número de ordenadores por cada 100 habitantes es otro de los indicadores tecnológicos analizado en 2009 para cada país. Los países con renta más elevada son también los que presentan un mayor número de ordenadores por habitante, con Bahrein (con 74,58 ordenadores por cada 100 habitantes) y Arabia Saudí (con $68,25)$ a la cabeza. Por otro lado, los países de renta media y baja tienen este índice muy pobre, oscilando para las rentas medias entre los 10,18 ordenadores por cada 100 habitantes de Líbano y los 0,77 de Iraq (sin duda, por la guerra sufrida y el desorden que conllevó), y para las rentas bajas, entre los 10,71 de Sudán y los 0,88 de Comores (debido a su analfabetismo y su escasa renta per cápita). Un caso digno de mención es de Argelia, puesto que a pesar de 
tener un nivel medio de renta, apenas si tiene 1 ordenador por cada 100 habitantes $(1,07)$.

Con respecto al número de ordenadores por cada 100 habitantes en función de la renta per cápita, como se ve en la figura 3, se aprecia nuevamente la separación de los países en función de su renta per cápita. Entre los países del grupo con rentas altas destacan de forma especial el caso de Bahrein $(\mathrm{BH})$ y Arabia Saudí (SA), posicionados muy por encima de la línea de tendencia. En el lado opuesto, por debajo de la tendencia están Qatar (QA) y Kuwait (KW). En el caso de las rentas intermedias, cinco países se sitúan por encima de la línea de tendencia pero próxima a ella, Líbano (LB), Túnez (TN), Siria (SY), Jordania (JO) y Marruecos (MA) y los otros cuatro lo hacen por debajo de la línea de tendencia, con especial mención a Libia (LY), Argelia (DZ) e Iraq (IQ) por su alejamiento de la misma. Por último, se halla el grupo de los países con rentas más bajas, donde destaca especialmente Sudán (SD) que sobrepasa considerablemente la media y, en sentido contrario, Comores (KM), que se sitúa por debajo de la línea de tendencia y a cierta distancia de ella. Somalia no aparece en la distribución por no disponer de datos económicos en el año 2008.

Si se analiza la evolución sufrida por los Estados árabes desde 2010 hasta 2013 teniendo en cuenta el porcentaje de ordenadores por hogar, se obtiene un incremento del $20,32 \%: 28,3 \%$ en $2010,31,7 \%$ en $2011,34 \%$ en 2012 , y sin datos en 2013.

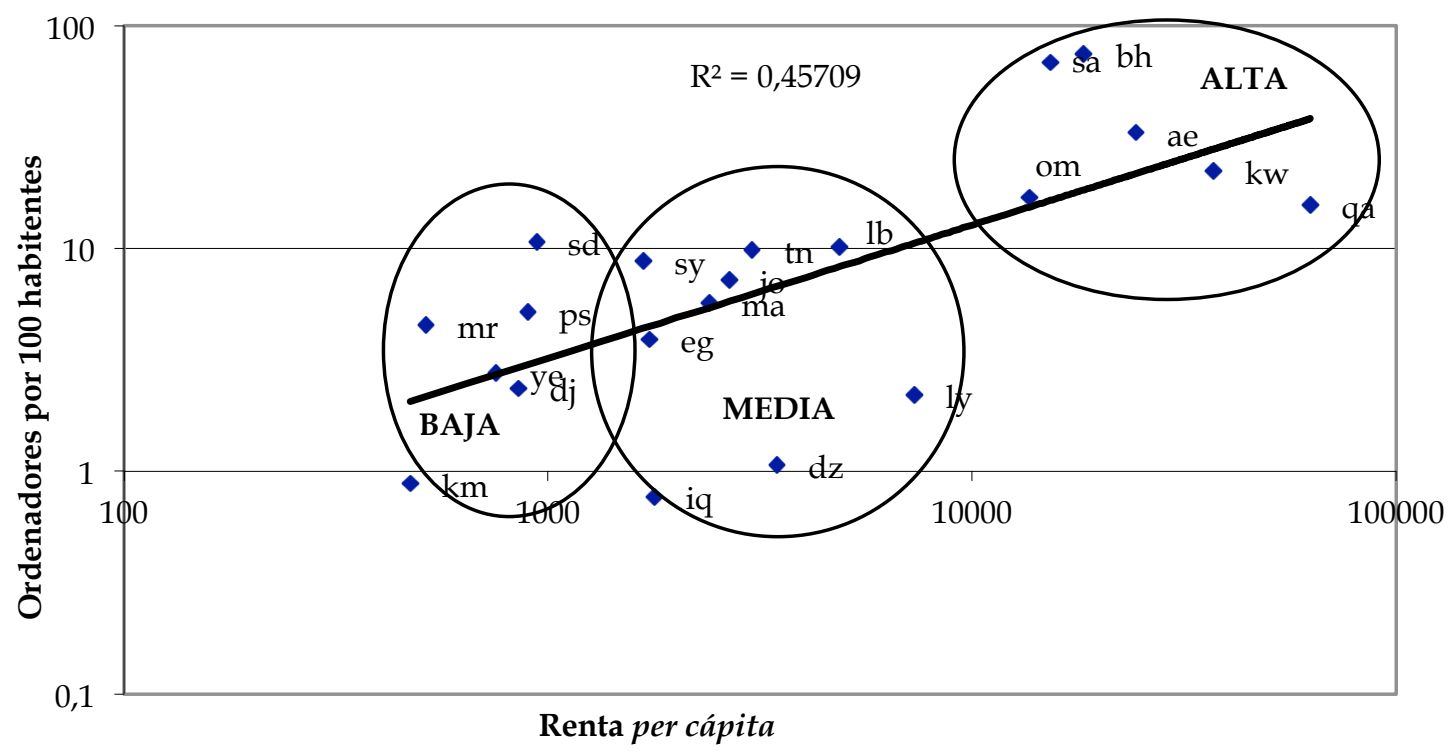

Figura 3. Ordenadores por cada 100 habitantes en 2009 en función de la renta per cápita en el año 2008

\subsubsection{Internet}

Hoy en día, el uso de Internet en un país es un indicador fundamental para comprobar el estado de su tecnología. En el mundo árabe, el uso de Internet es generalmente inferior a la media mundial. Este hecho está condicionado por factores externos (embargos, guerras, inestabilidad política, etc.), pero también por causas internas relacionadas, en gran medida, con las prohibiciones que los propios gobiernos de los países árabes imponen a sus habitantes. En este sentido, muchos países han introducido medidas para controlar las actividades de Internet, ya sea promulgando leyes al respecto (como los Emiratos Árabes Unidos (UAE Cyber Crimes Law, 02/11/2007); o Arabia Saudí (Westley, 26/01/2008)), instalando sistemas de vigilancia que permiten controlar los hábitos de navegación de los usuarios, especialmente en establecimientos como los cibercafés -este es el caso, por ejemplo, de Argelia (Borowianh, 01/05/ 2007), Jordania (Jordan: New restrictions..., 11/03/2008); Kuwait (Kuwait: Bureau of democracy..., 11/03/2008); Siria (Oweis, 13/03/2008); o Egipto (Noman, 16/04/2009)_; o poniendo en práctica sistemas de seguridad en la Red para bloquear el acceso a contenidos en línea específicos, sobre todo, si se trata de información de seguridad nacional o relacionada con la moral pública - como en el caso de Iraq (AbdulRahman, 09/02/2009)—.

En definitiva, los censores de los países árabes, en el intento de controlar el contenido político de Internet, utilizan técnicas de filtrado, promulgan 
leyes y reglamentos, usan la vigilancia y el control, restricciones físicas, el acoso y las detenciones extrajudiciales. El filtrado de contenido considerado ofensivo por razones religiosas, morales y culturales es un fenómeno generalizado en muchos países. Sin embargo, pese a la utilización de estas prácticas de censura que crean un clima de miedo que persigue acallar a los disidentes en línea, las redes sociales han hecho posible que en 2011, miles y miles de internautas de dichos países se hayan levantado para clamar por mayor libertad (como es el caso de Siria o Egipto).

Relacionado con el control y la vigilancia tecnológica en Internet se encuentra el sector al que pertenecen las telecomunicaciones en la mayoría de los países de la zona MENA en el año 2008. En esta fecha, la mayor parte de los países tenían acceso a las telecomunicaciones a través del Estado (Arabia Saudí, Argelia, Comores, Dijbouti, Emiratos Árabes, Kwait, Líbano, Libia, Palestina, Qatar, Siria y Yemen), solamente dos tenían el sector de las telecomunicaciones en manos privadas (Somalia y Jordania) y el resto pertenecían al sector parcialmente privado (Bahrein, Egipto, Marruecos, Mauritania, Omán, Sudán y Túnez). En este caso no se dispone de datos sobre Iraq debido a su estado de guerra en 2008). A este respecto, de la lectura rápida de las noticias de prensa sobre el estado de Internet en la región, se desprende que los gobiernos en Oriente Medio y en África, a la vez que invierten en tecnologías para la censura, también lo hacen en los medios de comunicación y proyectos de tecnologías de la información apoyados, en muchos casos, por empresas occidentales del sector de las tecnologías de la información que les ayudan a construir la infraestructura necesaria para el desarrollo en la sociedad de la información (Arfaoui, 01/08/2008).

A pesar del crecimiento esperado del número de usuarios conectados a Internet debido al avance de las tecnologías, el ciudadano encuentra, además de los políticos, una serie de obstáculos, económicos o administrativos para usar la Red, como, por ejemplo, el escaso porcentaje de información disponible en Internet en lengua árabe, que es todavía muy inferior al utilizado en otros idiomas. Como prueba de ello, esta variable se ha analizado en el conjunto de los 268 sitios web de universidades de la zona MENA objeto de estudio de los indicadores webmétricos en 2009, y se ha obtenido que la mayoría de los sitios permiten la consulta en dos o tres lenguas: inglés, francés y árabe. El 60,07\% de los web universitarios se pueden consultar en inglés (161 de 268), el 55,22\% en lengua árabe
(148 de 268) y el $25,37 \%$ en lengua francesa (68 de 268). Por un lado, el alto porcentaje de universidades en lengua inglesa denota la apertura del mundo árabe hacia el exterior, pero por otro, el que solo el $55,22 \%$ de ellas estén en árabe corrobora el hecho de que la información en Internet en esta lengua es todavía escasa. Ante esta situación, algunos países árabes han tenido iniciativas para aumentar el contenido de las páginas web en árabe. Este es el caso de Qatar, donde Microsoft está trabajando en tecnologías para la traducción que proporcionen a los usuarios de esta lengua un mayor acceso a Internet.

En resumen, la evolución general experimentada por los Estados Árabes desde 2010 hasta 2013 con respecto a diversos indicadores relacionados con la tecnología asociada a Internet ha sido muy positiva. Así, por cada 100 habitantes, el incremento de 2013 respecto a 2010 en suscripciones a Internet por banda ancha fija (cable) ha sido del 50,22\%: 2010, 1,9; 2011, 2,2; 2012, 2,6; y 2103, 2,8. Un incremento que se ha disparado en el caso de suscripciones a Internet por banda ancha móvil en un 305\%: 2010, 5,1; $2011,13,1 ; 2012,16,1$; y $2013,20,6$.

El porcentaje de hogares y de personas con acceso a Internet, también se ha visto favorecido con el paso de los años incrementándose, respectivamente, en un $37,83 \%-2010,24,4 \%$; $2011,28,8 \%$; 2012, 31,2\%; y 2013, 33,7\%-y un $62,57 \%$ en 2013 con respecto a 2010: 2010, $23 \%$; 2011, 26,6\%; 2012, 33,8\%; y 2013 , 37,4\%.

\subsection{Ranking web de las universidades} árabes de la zona MENA

Los indicadores tecnológicos han mostrado que la renta per cápita de los países árabes influye en el acceso y utilización de la tecnología de la información y la comunicación y, por consiguiente, en la presencia de los países en Internet. Los indicadores webmétricos permiten comprobar, además, la calidad de dicha presencia en la Red. Para ello, en esta investigación se analiza la presencia y calidad de los sitios web universitarios (268) de la zona MENA aplicando sobre ellos los 16 indicadores webmétricos explicados en la metodología.

En la tabla II se puede ver el número y la proporción de universidades que tiene cada uno de los países que forman parte del estudio en 2009, además del tipo de renta y la población de cada país. Hay que recordar que, aunque son 22 los países de la zona MENA, las Islas Comores no participan en esta parte de la investigación porque no tienen ninguna universidad con sitio web, por eso, aunque se aprecian 22 frac- 
ciones en la tabla, una de ellas corresponde al apartado denominado Varios (ya que existe una universidad que pertenece a varios países Arab Open University).

\begin{tabular}{lcrrr}
\hline País & $\begin{array}{r}\text { Renta } \\
\text { (2008) }\end{array}$ & $\begin{array}{r}\text { Población } \\
\text { (millones) }\end{array}$ & \multicolumn{1}{c}{$N^{\circ}$} & $\%$ \\
\hline Egipto & Media & 76,84 & 31 & 11,57 \\
\hline Argelia & Media & 34,37 & 25 & 9,33 \\
\hline Arabia Saudí & Alta & 25,29 & 24 & 8,96 \\
\hline Jordania & Media & 6,12 & 21 & 7,84 \\
\hline Túnez & Media & 10,44 & 20 & 7,46 \\
\hline Líbano & Media & 4,14 & 19 & 7,09 \\
\hline EAU & Alta & 4,5 & 15 & 5,60 \\
\hline Sudán & Baja & 39,88 & 15 & 5,60 \\
\hline Marruecos & Media & 31,61 & 14 & 5,22 \\
\hline Yemen & Baja & 23,07 & 13 & 4,85 \\
\hline Siria & Media & 20,45 & 12 & 4,48 \\
\hline Palestina & Baja & 4,15 & 11 & 4,10 \\
\hline Iraq & Media & 29,49 & 10 & 3,73 \\
\hline Bahrein & Alta & 0,77 & 8 & 2,99 \\
\hline Libia & Media & 6,28 & 8 & 2,99 \\
\hline Somalia & Baja & 8,96 & 6 & 2,24 \\
\hline Kuwait & Alta & 2,92 & 4 & 1,49 \\
\hline Omán & Alta & 2,65 & 4 & 1,49 \\
\hline Qatar & Alta & 0,86 & 4 & 1,49 \\
\hline Mauritania & Baja & 3,2 & 2 & 0,75 \\
\hline Dijbuti & Baja & 0,85 & 1 & 0,37 \\
\hline Varios & & & 1 & 0,37 \\
\hline Total & & & 268 & 100 \\
\hline & & & \\
\hline Tahla & & & \\
\hline
\end{tabular}

Tabla II. Número de universidades con web por países en 2009

Como se aprecia en la tabla II, en el caso concreto de las universidades, no hay relación entre la renta per cápita de los países y el porcentaje de sitios web universitarios que tienen. Es decir, la renta de un país no es indicativa de la representación de sus universidades en Internet. Así, por ejemplo, Kuwait, Omán o Qatar, con rentas altas, tienen un porcentaje muy escaso de presencia en la Red a través de sus universidades; en cambio, Sudán y Yemen, con rentas bajas se sitúan en la zona intermedia-superior de la tabla. Lógicamente, donde sí existe una correlación elevada $(r=0,7)$ es entre la población de cada país y la proporción de sitios web universitarios que tienen. Es decir, la población de los países árabes influye en la presencia de sus universidades en la Red.
Para comprobar la relación entre la cantidad de universidades de cada país presentes en Internet y la calidad de las mismas, y ver su relación con la renta per cápita de cada país, se analiza el comportamiento de las 268 universidades de la zona MENA teniendo en cuenta los 16 indicadores webmétricos que incluyen (una vez que éstos han sido ponderados tal y como se ha descrito en la metodología). Se obtiene así un ranking web de las universidades atendiendo a la calidad de los indicadores que contiene cada una de ellas.

El Apéndice, ordenado descendentemente por la columna Peso, muestra el peso final (desde 32,65 hasta 0,45 ) que obtiene cada una de las 268 universidades árabes tras aplicar la ponderación de sus indicadores. El Apéndice está dividido en cuatro Áreas (I, II, III y IV) de 67 universidades cada una, que representan la posición o el ranking web obtenido por cada universidad según el método señalado. Los sitios web del Área I son los que han obtenido los mayores pesos y, por consiguiente, las mejores posiciones según el método usado, seguidos de las universidades del Área II, Área III y Área IV. Se puede observar en el Área III que una universidad está adjudicada al país Varios lo que significa que pertenece a varios países. Para comprobar la evolución sufrida por algunas de las universidades con respecto a 2007, se puede consultar el trabajo de Al-Dwairi, FabaPérez y Vargas-Quesada (2010).

La figura 4 muestra la relación entre la renta per cápita de los países y la calidad o posición en el ranking obtenido por sus universidades según su Peso. Así, en valores porcentuales en relación al total de sitios web en cada Área, la figura 4 muestra que en las cuatro Áreas la renta media es la que alcanza los mayores porcentajes (aspecto que no resulta demasiado extraño, ya que el $60 \%$ de las universidades pertenecen a esta categoría). Lo que sí es preciso examinar con mayor precisión es la renta que sigue a la media en las distintas Áreas: la renta media es seguida por la renta alta en las Áreas I $(23,88 \%)$ y II $(34,33 \%)$ y por la renta baja en las Áreas III $(21,21 \%)$ y IV $(23,88 \%)$. Es decir, en las Áreas I y II, donde se hallan las universidades árabes mejor clasificadas según su peso, existe una mayor proporción de universidades con renta per cápita media-alta (en el Área I suman el $82,09 \%$ y en el Área II suman el 91,05\%). Asimismo, en las Áreas III y IV, donde se hallan las universidades árabes peor clasificadas, o con pesos más pequeños, existe una mayor proporción de universidades con renta per cápita media-baja (en el Área III suman el $87,88 \%$ y el Área IV el 82,09\%). 


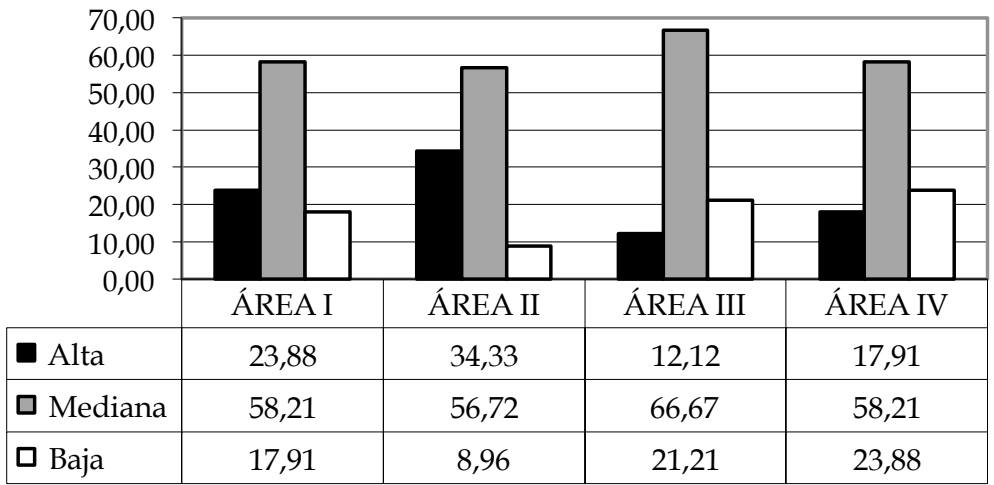

Fig. 4. Porcentaje de webs universitarias en cada área en relación a la renta per cápita de su país

\section{Conclusiones}

Los resultados obtenidos y su posterior interpretación nos han conducido a las siguientes conclusiones que corroboran la hipótesis de partida.

Apenas hay estudios sobre los países árabes en Internet, por lo que este trabajo permite conocer la presencia que el mundo árabe y sus universidades tenían en Internet en el año 2009, así como la evolución general sufrida (favorable) por los Estados Árabes durante el periodo 20102103 con respecto a su tecnología. Para ello, se ha aplicado una metodología extrapolable a cualquier entorno geográfico, comprobándose su operatividad en los países árabes y en sus universidades.

Se ha demostrado que la situación económica de los países árabes en el período estudiado influye notablemente en el nivel del servicio que proporcionan las TIC en los mismos. Gran parte de los países de la región tienen un sistema económico centralizado, especialmente en las áreas de infraestructura y servicios, lo que produce como consecuencia la eliminación de la competencia y, por tanto, del desarrollo en términos generales.

El nivel de desarrollo de las TIC depende en gran medida de la renta per cápita del país existiendo una relación directa entre la difusión del servicio telefónico y el nivel de la renta por habitante.

El desarrollo de Internet como fuente de información afronta, además, otros problemas relacionados con el propio idioma árabe dentro de la red (no hay suficientes recursos en esta lengua), o con la gran subida de precios generalizada de los ordenadores y la dificultad de su uso por parte de los usuarios.

La difusión de Internet en la zona MENA se relaciona con el nivel económico, pero también con la apertura social y cultural de los países, con el papel que desempeñan los Estados en impulsar la difusión del servicio, así como con la toma de conciencia y la creación de un ambiente favorable para el desarrollo de la Red.

El factor económico de los países influye en la posición que los sitios web de sus universidades obtienen en el ranking de calidad hallado con el análisis de sus indicadores webmétricos. Se ha comprobado que en las Áreas I y II, donde se encuentran las universidades árabes mejor clasificadas, existe una mayor proporción de universidades con renta per cápita media-alta, mientras que en las Áreas III y IV, donde se hallan las universidades árabes peor clasificadas, existe una mayor proporción de universidades con renta per cápita media-baja.

En este sentido, se puede decir que el factor económico de los países de la zona MENA, influye positivamente en la construcción y el diseño de los sitios web de sus universidades, ya que disponen de mayores posibilidades de inversión. Sin embargo, hay que matizar que no es un factor directamente proporcional a la posición en el ranking, ya que en el caso de las universidades con renta per cápita alta la mayor proporción de las mismas se hallan en el Área II, seguida de las Áreas I, IV y III en este orden $y$, en el caso de los sitios universitarios con renta baja, la mayoría de los mismos se sitúan en el Área IV, seguida de las Áreas III, I y II. Es decir, además del factor económico, otras condiciones sociopolíticas estructurales también influyen en la posición en el ranking.

\section{Agradecimientos}

Este trabajo ha sido financiado por la Junta de Extremadura, Consejería de Empleo, Empresa e Innovación y el Fondo Social Europeo como parte de la ayuda a grupos GR10019.

\section{Referencias}

Abdul-Rahman, F. (2009). عبد الرحمن, فاروق عبد القادر. [El Ministerio de Comunicaciones firmó un contrato para manejar las brechas de seguridad a la Internet en Iraq].

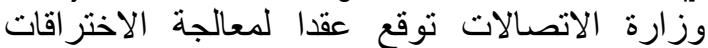

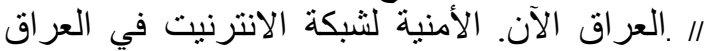
[Iraq ahora]. 09/02/2009. http://www.iraqalaan.com/ $(11 / 12 / 2009)$.

Abrizah, A.; Noorhidawati, A.; Kiran, K. (2010). Global visibility of Asian universities' Open Access institutional repositories. // Malaysian Journal of Library \& Information Science. 15:3, 53-73.

Alawi, Hind (2006). علوي, [Los indicadores que miden la sociedad de la información: una visión Mentori bibliotecarios de la Universidad de Constantine-

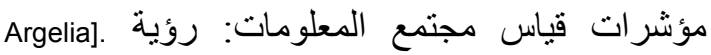
Cybrarians Journal. 10 . http://journal.cybrarians.info/ $(21 / 11 / 2009)$ 
Al-Dwairi, K.; Faba-Pérez, C.; Vargas-Quesada, B. (2010). Indicadores Webmétricos de carácter Formal para Evaluar el Posicionamiento de las Universidades: el caso de los Países Árabes. // Investigación Bibliotecológica: archivonomía, bibliotecología e información. 24:52, 145171.

Al-Dwairi, K.; Herrero-Solana (2007). La sociedad de la información en los países: una aproximación al análisis de indicadores socioeconómicos. // Investigación Bibliotecológica: archivonomía, bibliotecología e información. $21: 43,185-208$

Almind, T.C.; Ingwersen, P. (1997). Informetric analyses on the World Wide Web: A methodological approach to Webmetrics. // Journal of Documentation. 53:4, 404-426.

Al-Mobarek, S. (2008). صبرى الحاج, المبارك. [La información y su papel en el desarrollo]. المعلومات ودور هـاجيا

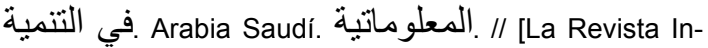
formática]. 25, 2008. http://informatics.gov.sa (02/12/2009).

Arakaki, M.; Willett (2009). Webometric analysis of departments of librarianship and information science: a followup study. // Journal of Information Science. 35:2, 143152.

Arfaoui, J. (2008). Internet chat rooms offer romance to Maghreb residents. // Magharebia. 01/08/2008. http://www.magharebia.com/cocoon/awi/xhtml1/en_GB/f eatures/awi/reportage/2008/08/01/reportage-01 (01/12/2009).

Asadi, M.; Shekofteh, M. (2009). The relationship between the research activity of Iranian medical universities and their web impact factor, // The Electronic Library. 27, n.6, 1026-1043.

Association of Arab Universities, Jordan. http://www.aaru.edu.jo/.

Borowianh, F. (2007). فتحية, البورون.]Argelia: Seguridad declara la guerra a los cafés, "Internet" para frustrar pro-

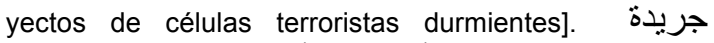
الرياض : الجزائر: أجهزة الأمن تعلن الحرب على مقاهي "الانترنت" لإحباط مشاريع إنز خلايا إرهابية نائمة 01/05/2007 http://www.alriyadh.com/2007/05/01/article246175.html (13/07/2009).

Caballero-Cortés, L.; Faba-Pérez, C.; Moya-Anegón, F. (2009). Evaluación comparativa de la accesibilidad de los espacios Web de las bibliotecas universitarias españolas y norteamericanas. // Investigación Bibliotecológica: archivonomía, bibliotecología e información. 47:23, 45-66.

Chung, Y. M. [et al.] ( ). Characteristics and link structure of a national scholarly Web space: The case of South Korea. // Scientometrics. 80:3, 597-614, 2009.

Elgohary, A. (2008). The Arab universities on the web: a webometric study. // Electronic Library. 26:3, 374-386.

Faba-Pérez, C.; Guerrero-Bote. P.; Moya-Anegón, F. (2005). Self-organizing maps of Web spaces based on formal characteristics. // Information Processing and Management. 41:2, 331-346.

Farrag, A. R. (2006). فراج, عبد الرحمن عبد الهادي . [La presencia electrónica de las universidades saudíes en Internet: un estudio acerca del Factor Impacto Web]. الحضور الإلكتروني للجامعات السعودية على الثبكة

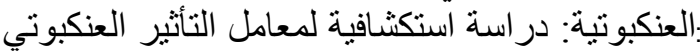

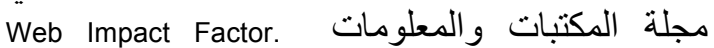

. Arab Journal of Library \& Information Science. 26:3, 151-174.

International Telecommunication http://www.itu.int/ar/pages/default.aspx.

Jordan: New restrictions on Internet cafés and violating privacy of users. // The Arabic Network for Human Rights Information. Egypt. 11/03/2008. http://anhri.net/en/reports/2008/pr0311.shtml (24/11/2009).

Kuwait: Bureau of Democracy, Human Rights, and Labor (2009). Released by the Bureau of Democracy, Human Rights, and Labor. 11/03/2008. http://www.state.gov/g/drl/rls/hrrpt/2007/100599.htm $(12 / 12 / 2009)$

Liga de estados árabes. http://www.arabe.galeon.com/ppaiseslea.htm, y http://www.arableagueonline.org/las/index.jsp

Moscovkin. M. (2010). The Webometric Estimate of the Publication Activities of Universities: The Influence of the Belgorod Declaration. // Scientific and Technical Information Processing. 37:1, 49-54.

Noman, H. (2009). Restriction on Internet use in the Middle East on the rise: Internet cafés in Saudi must install hidden cameras. // OpenNet Initiative. 16/04/2009. http://opennet.net/blog/2009/04/restriction-internet-usemiddle-east-rise-internet-caf\%C3\%A9s-saudi-mustinstall-hidden-came (11/12/2009).

Noruzi, A. (2006). Web Presence and Impact Factors for Middle-Eastern Countries. // Online. 30:2, 22-28.

Nwagwu, W. E.; Agarin, O. (2008). Nigerian university websites: A webometric analysis. // Webology. 5:4. http://www.webology.org/2008/v5n4/a65.html (01/04/2012).

Ortega, J. L.; Aguillo, I. (2008a). Linking patterns in the European Union's Countries: geographical maps of the European academic web space. // Journal of Information Science. 34:5, 705-714.

Ortega, J. L.; Aguillo, I. (2008b). Visualization of the Nordic academic web: Link analysis using social network tools. // Information Processing and Management. 44, 16241633.

Ortega, J. L.; Aguillo, I. (2009a). Mapping world-class universities on the web. // Information Processing and Management. 45:2, 272-279.

Ortega, J. L.; Aguillo, I. (2009b). Análisis estructural de la web académica iberoamericana. // Revista española de Documentación Científica. 32:3, 51-64.

Oweis, K. Y. (2008). Syria expands 'iron censorship' over Internet," Reuters. // Thomson Reuters. 13/03/2008. http://uk.reuters.com/article/2008/03/13/oukin-uk-syriainternet-idUKL138353620080313?sp=true (02/11/2012).

Payne:, Thelwall, M. (2004). A Statistical Analysis of UK Academic Web Links. // Cybermetrics. 8:1.

Payne:, Thelwall, M. (2008a). Longitudinal trends in academic web links. // Journal of Information Science. 34:1, 314.

Payne:, Thelwall, M. (2008b). Do academic link types change over time?. // Journal of Documentation. $64, \mathrm{n} 5$, 707-720.

Smith, A.G.; Thelwall, M. (2002). Web Impact Factors for Australasian universities. // Scientometrics. 54:1-2, 363380.

Tang, R.; Thelwall, M. (2002). Exploring the pattern of links between Chinese university Web Sites. // American Society for Information Science and Technology. v.39:1, 417-424. 
Tang, R.; Thelwall, M. (2003). U.S. academic departmental Web-site interlinking in the United States Disciplinary differences. // Library \& Information Science Research. 25, 437-458.

UAE cyber crimes law. Gulf News Report. 02/11/2007. http://archive.gulfnews.com/uae/uaessentials/more_stori es/10018507.html (24/08/2009).

Universities Worldwide. http://univ.cc/world.php

Vargas-Quesada, B.; Al-Dwairi, K.; Faba-Pérez, C.; MoyaAnegón. (2013). Web Structure and Influence of the Arab Universities of the MENA Zone (Middle East and North Africa): visualization and Analysis. // Aslib Proceedings. 65:6, 623-643.
Westley, D. (2008). Saudi Tightens Grip on Internet Use. Arabian Business. 26/01/2008. http://www.arabianbusiness.com/saudi-tightens-grip-oninternet-use-192173.html (20/12/2012).

Wilkinson, D. [et al.]. (2003). Motivations for academic web site interlinking: evidence for the Web as a novel source of information on informal scholarly Communications. // Journal of Information Science. 29:1, 49-56.

Enviado: 2014-02-02. Segunda versión: 2014-06-13. Aceptado: 2014-07-11.

\section{Apéndice. Peso final de los sitios web universitarios de la zona MENA en 2009}

\begin{tabular}{|c|c|c|c|}
\hline ÁREA & & & \\
\hline Pais & Nombre de la Universidad & URL & Peso \\
\hline SY & Private University of Science \& Arts & http://www.pusa-sy.org/ & 32,65 \\
\hline sY & $\begin{array}{c}\text { Wadi International Private } \\
\text { University }\end{array}$ & http://www.wgsu.biz/ & 30,81 \\
\hline $\mathrm{AE}$ & University of Sharjah & http://www.sharjah.ac.ae/ & 29,92 \\
\hline $\mathrm{BH}$ & Arabian Gulf University & http://www.agu.edu.bh/ & 29,76 \\
\hline QA & Carnegie Mellon University in Qatar & http://www.qatar.cmu.edu/ & 29,65 \\
\hline JO & Al-Balqa Applied University & http://www.bau.edu.jo/ & 29,52 \\
\hline PS & An-Najah National University & http://www.najah.edu/ & 28,64 \\
\hline $\mathrm{DZ}$ & Université d'Alger & http://www.univ-alger.dz/ & 28,39 \\
\hline YE & Saba University & http://www.sabauni.net/ & 28,13 \\
\hline DZ & Université de Bejaia & http://www.univ-bejaia.dz/ & 27,19 \\
\hline MA & Université Hassan II Casablanca & http://www.uh2c.ac.ma/ & 27,09 \\
\hline MA & Université Abdelmalek Essadi & http://www.uae.ma/ & 26,92 \\
\hline SA & $\begin{array}{l}\text { Imam Muhammad ibn Saud Islamic } \\
\text { University }\end{array}$ & http://www.imamu.edu.sa/ & 26,06 \\
\hline EG & Mansoura University & http://www.mans.edu.eg/ & 25,63 \\
\hline LB & Université Saint-Joseph & http://www.usj.edu.lb/ & 25,1 \\
\hline PS & Birzeit University & http://www.birzeit.edu/ & 25,1 \\
\hline SD & Open University of Sudan & http://www.ous.edu.sd/ & 24,81 \\
\hline LB & University of Balamand & http://www.balamand.edu.lb/ & 24,8 \\
\hline SA & Umm Al-Qura University & http://www.uqu.edu.sa/ & 24,78 \\
\hline SA & $\begin{array}{l}\text { King Fahd University of Petroleum } \\
\text { and Minerals }\end{array}$ & http://www.kfupm.edu.sa/ & 24,76 \\
\hline $\mathrm{AE}$ & American University of Sharjah & http://www.aus.edu/ & 24,52 \\
\hline EG & South Valley University & http://www.svu.edu.eg/ & 24,52 \\
\hline $\mathrm{DZ}$ & $\begin{array}{c}\text { Université de la Formation } \\
\text { Continue }\end{array}$ & http://www.ufc.dz/ & 24,22 \\
\hline SD & $\begin{array}{c}\text { Sudan University of Science \& } \\
\text { Technology }\end{array}$ & http://www.sustech.edu/ & 24,22 \\
\hline JO & Yarmouk University & http://www.yu.edu.jo/ & 23,98 \\
\hline SY & Al-Baath University & http://www.albaath-univ.edu.sy/ & 23,84 \\
\hline PS & Islamic University of Gaza & http://www.iugaza.edu.ps/ & 23,65 \\
\hline $\mathrm{AE}$ & University of Wollongong in Dubai & http://www.uowdubai.ac.ae/ & 23,32 \\
\hline IQ & Wassit University & http://www.uowasit.edu.iq/ & 23,25 \\
\hline SA & King Faisal University & http://www.kfu.edu.sa/ & 23,22 \\
\hline SA & Islamic University of AI Madinah & http://www.iu.edu.sa/ & 23,15 \\
\hline SD & University of Juba & http://www.juba.edu.sd/ & 23,08 \\
\hline MA & Université Hassan 1er & http://www.uh1.ac.ma/ & 22,92 \\
\hline EG & Assiut University & http://www.aun.edu.eg/ & 22,72 \\
\hline DZ & Université Ferhat Abbas Sétif & http://www.univ-setif.dz/ & 22,49 \\
\hline SA & $\begin{array}{l}\text { Naif Arab University for Security } \\
\text { Sciences }\end{array}$ & http://www.nauss.edu.sa/ & 22,43 \\
\hline $\mathrm{AE}$ & $\begin{array}{c}\text { Khalifa University of Science, } \\
\text { Technology and Research }\end{array}$ & http://www.ku.ac.ae/ & 21,8 \\
\hline
\end{tabular}

\begin{tabular}{|c|c|c|c|}
\hline $\mathrm{AE}$ & Zayed University & http://www.zu.ac.ae/ & 21,79 \\
\hline PS & Bethlehem University & http://www.bethlehem.edu/ & 21,67 \\
\hline JO & Philadelphia University & http://www.philadelphia.edu.jo/ & 21,66 \\
\hline EG & American University in Cairo & http://www.aucegypt.edu/ & 21,64 \\
\hline SA & King $\begin{array}{r}\text { Abdullah } \\
\text { University }\end{array}$ Bin Abdulaziz & http://www.kau.edu.sa/ & 21,6 \\
\hline SD & University of Khartoum & http://www.uofk.edu/ & 21,11 \\
\hline LB & Université Saint-Esprit de Kaslik & http://www.usek.edu.lb/ & 21,1 \\
\hline $\mathrm{AE}$ & $\begin{array}{l}\text { Ajman University of Science and } \\
\text { Technology }\end{array}$ & http://www.ajman.ac.ae/ & 20,97 \\
\hline sY & Tishreen University & http://www.tishreen.shern.net/ & 20,97 \\
\hline PS & Al-Quds University & http://www.alquds.edu/ & 20,92 \\
\hline TN & University of Sousse & http://www.uc.rnu.tn/ & 20,75 \\
\hline JO & Zarqa Private University & http://www.zpu.edu.jo/ & 20,67 \\
\hline YE & Al-iman University & http://www.jameataleman.org/ & 20,53 \\
\hline TN & Université Centrale & http://www.universitecentrale.net & 20,4 \\
\hline MA & $\begin{array}{l}\text { Université Sidi Mohamed Ben } \\
\text { Abdellah }\end{array}$ & http://www.usmba.ac.ma/ & 20,37 \\
\hline SA & Prince Mohammad University & http://www.pmu.edu.sa/ & 20,28 \\
\hline MA & Université Mohammed V-Agdal & http://www.um5a.ac.ma/ & 20,25 \\
\hline MA & Al Akhawayn University & http://www.aui.ma/ & 19,91 \\
\hline LB & Université Sagesse & http://www.uls.edu.lb/ & 19,87 \\
\hline sY & Arab International University & http://www.aiu.edu.sy/ & 19,84 \\
\hline DZ & Université Amar Telidji Laghouat & http://www.lagh-univ.dz/ & 19,79 \\
\hline PS & Al-Quds Open University & http://www.qou.edu/ & 19,75 \\
\hline LY & Al-Fateh University & http://www.alfateh.edu.ly/ & 19,64 \\
\hline EG & $\begin{array}{c}\text { Modern Sciences and Arts } \\
\text { University }\end{array}$ & http://www.msa.eun.eg/ & 19,6 \\
\hline LB & Lebanese American University & http://www.lau.edu.lb/ & 19,48 \\
\hline TN & Université Virtuelle de Tunis & http://www.uvt.rnu.tn/ & 19,46 \\
\hline LB & American University of Beirut & http://www.aub.edu.lb/ & 19,42 \\
\hline LY & Al-Tahadi University & http://www.altahadi.edu.ly/ & 19,41 \\
\hline MA & Université Cadi Ayyad & http://www.ucam.ac.ma/ & 19,25 \\
\hline JO & Mu'tah University & http://www.mutah.edu.jo/ & 19,09 \\
\hline \multicolumn{4}{|c|}{ ÁREA II } \\
\hline JO & Tafila Technical University & http://www.ttu.edu.jo/ & 19,05 \\
\hline SA & King Khalid University & http://www.kku.edu.sa/ & 19,04 \\
\hline EG & Menofia University & http://www.menofia.edu.eg/ & 18,94 \\
\hline EG & Université Française d'Egypte & http://www.ufe.edu.eg/ & 18,9 \\
\hline IQ & Babylon University & http://www.babylon-uni.com/ & 18,88 \\
\hline DZ & Université Abou Bekr Belkaid & http://www.univ-tlemcen.dz/ & 18,85 \\
\hline JO & Al-Hussein Bin Talal University & http://www.ahu.edu.jo/ & 18,85 \\
\hline SA & Prince Sultan University & http://www.psu.edu.sa/ & 18,75 \\
\hline PS & El-Azhr University Gaza & http://www.alazhar.edu.ps/ & 18,66 \\
\hline
\end{tabular}

Faba-Pérez, Cristina, Vargas-Quesada, Benjamín, Al-Dwairi, K. M. O., Moya-Anegón, Félix. La presencia de los países árabes en Internet: un análisis a través de sus indicadores tecnológicos y de sus universidades. // Ibersid. 8 (2014) 19-31. ISSN 1888-0967. 


\begin{tabular}{|c|c|c|c|}
\hline EG & Zagazig University & http://www.zu.edu.eg/ & 18,62 \\
\hline SA & Tabouk University & http://www.ut.edu.sa/ & 18,57 \\
\hline LB & Beirut Arab University & http://www.bau.edu.lb/ & 18,56 \\
\hline $\mathrm{DZ}$ & Université de Boumerdès & http://www.umbb.dz/ & 18,5 \\
\hline $\mathrm{AE}$ & UAE University & http://www.uaeu.ac.ae/ & 18,49 \\
\hline LB & Notre Dame University & http://www.ndu.edu.lb/ & 18,42 \\
\hline SD & International University of Africa & http://www.iua.edu.sd/ & 18,32 \\
\hline EG & Sinai University & http://www.su.edu.eg/ & 18,26 \\
\hline KW & $\begin{array}{l}\text { Gulf University for Science and } \\
\text { Technology }\end{array}$ & http://www.gust.edu.kw/ & 18,16 \\
\hline DZ & Université de Biskra & http://www.univ-biskra.dz/ & 18,12 \\
\hline MA & Université Mohammed V-Souissi & http://www.um5s.ac.ma/ & 18,07 \\
\hline DZ & Université Mohamed Boudiaf & http://www.univ-usto.dz/ & 18 \\
\hline EG & Pharos University in Alexandria & http://www.pua.edu.eg/ & 17,99 \\
\hline DZ & Université Hadj Lakhdar Batna & http://www.univ-batna.dz/ & 17,95 \\
\hline JO & Al al-Bayt University & http://www.aabu.edu.jo/ & 17,85 \\
\hline jO & German-Jordanian University & http://www.gju.edu.jo/ & 17,8 \\
\hline Dz & Université 08 Mai 1945 & http://www.univ-guelma.dz/ & 17,77 \\
\hline OM & Sohar University & http://www.soharuni.edu.om/ & 17,75 \\
\hline JO & Hashemite University & http://www.hu.edu.jo/ & 17,65 \\
\hline OM & Dhofar University & http://www.du.edu.om/ & 17,52 \\
\hline OM & Sultan Qaboos University & http://www.squ.edu.om/ & 17,51 \\
\hline YE & National University & http://www.nationaluni.net/ & 17,48 \\
\hline LB & Jinan University & http://www.jinan.edu.lb/ & 17,47 \\
\hline JO & $\begin{array}{c}\text { Princess Sumaya University for } \\
\text { Technology }\end{array}$ & http://www.psut.edu.jo/ & 17,46 \\
\hline SA & King Saud bin Abdullaziz University & http://www.ksau-hs.edu.sa/ & 17,46 \\
\hline QA & $\begin{array}{l}\text { Virginia Commonwealth University } \\
\text { in Qatar }\end{array}$ & http://www.qatar.vcu.edu/ & 17,45 \\
\hline DZ & Université Ibn Khaldoun & http://www.univ-tiaret.dz/ & 17,42 \\
\hline EG & Tanta University & http://www.tanta.edu.eg/ & 17,41 \\
\hline $\mathrm{DZ}$ & $\begin{array}{l}\text { Université des Sciences et de la } \\
\text { Technologie }\end{array}$ & http://www.usthb.dz/ & 17,4 \\
\hline $\mathrm{AE}$ & American University in Dubai & http://www.aud.edu/ & 17,35 \\
\hline IQ & University of Dohuk & http://www.dohukuni.net/ & 17,35 \\
\hline $\mathrm{BH}$ & Kingdom University & http://www.ku.edu.bh/ & 17,28 \\
\hline SA & Taibah University & http://www.taibahu.edu.sa/ & 17,23 \\
\hline QA & Qatar University & http://www.qu.edu.qa/ & 17,21 \\
\hline YE & $\begin{array}{l}\text { Yemeni University of Science and } \\
\text { Technology }\end{array}$ & http://www.ust.edu/ & 17,17 \\
\hline DZ & Université de Mostaganem & http://www.univ-mosta.dz/ & 17,06 \\
\hline PS & Hebron University & http://www.hebron.edu/ & 17 \\
\hline $\mathrm{AE}$ & Abu Dhabi University & http://www.adu.ac.ae/ & 16,84 \\
\hline SA & $\begin{array}{l}\text { King Abdullah University of Science } \\
\text { and Technology }\end{array}$ & http://www.kaust.edu.sa/ & 16,81 \\
\hline KW & American University of Kuwait & http://www.auk.edu.kw/ & 16,66 \\
\hline SA & King Saud University & http://www.ksu.edu.sa/ & 16,66 \\
\hline SA & Najran University & http://www.nu.edu.sa/ & 16,59 \\
\hline LY & University of Garyounis & http://www.garyounis.edu/ & 16,56 \\
\hline $\mathrm{BH}$ & University of Bahrain & http://www.uob.edu.bh/ & 16,54 \\
\hline $\mathrm{BH}$ & Medical University in Bahrain & http://www.rcsi-mub.com/ & 16,47 \\
\hline JO & University of Jordan & http://www.ju.edu.jo/ & 16,39 \\
\hline DZ & Université du 20 Août 1955 & http://www.univ-skikda.dz/ & 16,38 \\
\hline SA & Al-Baha university & http://www.bu.edu.sa/ & 16,31 \\
\hline $\mathrm{BH}$ & The Royal University for Women & http://www.ruw.edu.bh/ & 16,18 \\
\hline MR & Université de Nouakchott & http://www.univ-nkc.mr/ & 16,12 \\
\hline EG & Nile University & http://www.nileu.edu.eg/ & 16,07 \\
\hline LY & University of Seventh April & http://www.7aprilu.edu.ly/ & 16,04 \\
\hline JO & Al-Zaytoonah University of Jordan & http://www.alzaytoonah.edu.jo/ & 15,96 \\
\hline DZ & Université Kasdi Merbah Ouargla & http://www.ouargla-univ.dz/ & 15,75 \\
\hline EG & Helwan University & http://www.helwan.edu.eg/ & 15,75 \\
\hline
\end{tabular}

\begin{tabular}{|c|c|c|c|}
\hline EG & Ain Shams University & http://net.shams.edu.eg/ & 15,72 \\
\hline JO & AL-Isra Private University & http://www.isra.edu.jo/ & 15,68 \\
\hline $\mathrm{DZ}$ & Université de Jijel & http://www.univ-jijel.dz/ & 15,67 \\
\hline \multicolumn{4}{|c|}{ ÁREA III } \\
\hline EG & Benha University & http://www.benha-univ.edu.eg/ & 15,63 \\
\hline IQ & University of Mosul & http://www.uomosul.edu.iq/ & 15,63 \\
\hline JO & Applied Science Private University & http://www.asu.edu.jo/ & 15,62 \\
\hline $\mathrm{BH}$ & Gulf University & http://www.gulfuniversity.net/ & 15,58 \\
\hline $\mathrm{DZ}$ & Université d'Oran & http://www.univ-oran.dz/ & 15,56 \\
\hline IQ & University of Mustansiriyah & $\begin{array}{l}\text { http://www.uomustansiriyah.edu. } \\
\text { iq/ }\end{array}$ & 15,49 \\
\hline EG & Université Senghor d'Alexandrie & $\begin{array}{l}\text { http://www.usenghor- } \\
\text { francophonie.org/ }\end{array}$ & 15,44 \\
\hline OM & Nizwa University & http://www.unizwa.edu.om/ & 15,35 \\
\hline SY & Syrian Virtual University & http://www.svuonline.org/ & 15,32 \\
\hline PS & Al-Aqsa University & http://www.alaqsa.edu.ps/ & 15,31 \\
\hline MA & Université Hassan II-Mohammedia & http://www.univh2m.ac.ma/ & 15,22 \\
\hline sY & University of Kalamoon & http://www.uok.edu.sy/ & 15,12 \\
\hline $\mathrm{DZ}$ & Université Djillali Liabès & http://www.univ-sba.dz/ & 15 \\
\hline SD & Nile Valley University & http://www.nilevalley.edu.sd/ & 14,96 \\
\hline so & Mogadishu University & $\begin{array}{l}\text { http://www.mogadishuuniversity. } \\
\text { com/ }\end{array}$ & 14,84 \\
\hline EG & Suez Canal University & http://scuegypt.edu.eg/ & 14,83 \\
\hline EG & Future University & http://www.futureuniversity.info/ & 14,79 \\
\hline so & Puntland State University & $\begin{array}{l}\text { http://puntlandstateuniversity.co } \\
\mathrm{m} /\end{array}$ & 14,69 \\
\hline LY & Sebha University & http://www.sebhau.edu.ly/ & 14,65 \\
\hline $\mathrm{DZ}$ & Université Mentouri Constantine & http://www.umc.edu.dz/ & 14,61 \\
\hline EG & $\begin{array}{l}\text { Misr University for Science and } \\
\text { Technology }\end{array}$ & http://www.must.edu/ & 14,56 \\
\hline EG & Sohag University & http://www.sohag-univ.edu.eg/ & 14,54 \\
\hline YE & Yemen University & http://www.yemenuniversity.com & 14,46 \\
\hline MA & $\begin{array}{l}\text { Université Mohammed Premier } \\
\text { Oujda }\end{array}$ & http://www.univ-oujda.ac.ma/ & 14,43 \\
\hline KW & Kuwait University & http://www.kuniv.edu.kw/ & 14,4 \\
\hline JO & Irbid National University & http://www.inu.edu.jo/ & 14,35 \\
\hline EG & Cairo University & http://www.cu.edu.eg/ & 14,27 \\
\hline DZ & Université Saad Dahlab Blida & http://www.univ-blida.dz/ & 14,23 \\
\hline QA & Texas A\&M University at Qatar & http://www.qatar.tamu.edu/ & 14,23 \\
\hline AE & Alhosn University & http://www.alhosnu.ae/ & 13,9 \\
\hline EG & AL-Azhar University & http://www.azhar.edu.eg/ & 13,87 \\
\hline $\mathrm{AE}$ & Ittihad University & http://www.ittihad.ac.ae/ & 13,84 \\
\hline SD & Gezira University & http://www.uofg.edu.sd/ & 13,81 \\
\hline MA & Université lbn Tofaïl & http://www.univ-ibntofail.ac.ma/ & 13,78 \\
\hline LY & Omar Al-Mukhtar University & http://www.omu.edu.ly/ & 13,75 \\
\hline LB & Al Manar University of Tripoli & http://www.mut.edu.lb/ & 13,74 \\
\hline PS & Palestine Polytechnic University & http://www.ppu.edu/ & 13,74 \\
\hline TN & Université de Monastir & http://www.um.rnu.tn/ & 13,64 \\
\hline PS & Arab American University & http://www.aauj.edu/ & 13,58 \\
\hline SD & The National Ribat University & http://www.ribat.edu.sd/ & 13,54 \\
\hline TN & Université Libre de Tunis & http://www.ult-tunisie.com/ & 13,54 \\
\hline JO & $\begin{array}{l}\text { Amman Arab University for Higher } \\
\text { Studies }\end{array}$ & http://www.aau.edu.jo/ & 13,51 \\
\hline JO & Petra University & http://www.uop.edu.jo/ & 13,49 \\
\hline YE & Taiz University & http://www.taizun.net/ & 13,48 \\
\hline LB & Université Antonine & http://www.upa.edu.lb/ & 13,46 \\
\hline $\mathrm{AE}$ & $\begin{array}{l}\text { Al-Ain University of Science and } \\
\text { Technology }\end{array}$ & http://www.alainuniversity.ac.ae/ & 13,44 \\
\hline EG & Ahram Canadian University & http://www.acu.edu.eg/ & 13,43 \\
\hline YE & Alahgaff University & http://www.ahgaff.edu/ & 13,4 \\
\hline EG & British University in Egypt & http://www.bue.edu.eg/ & 13,34 \\
\hline SD & Shendi University & http://www.ush.sd/ & 13,33 \\
\hline
\end{tabular}




\begin{tabular}{|c|c|c|c|}
\hline IQ & University of Anbar & http://univ-anbar.org/ & 13,29 \\
\hline sy & $\begin{array}{l}\text { International University for Science } \\
\text { and Technology }\end{array}$ & http://www.iust.edu.sy/ & 13,29 \\
\hline LY & Open University & http://www.libopenuniv-edu.org/ & 13,11 \\
\hline DZ & $\begin{array}{l}\text { Université Mouloud Mammeri de } \\
\text { Tizi Ouzou }\end{array}$ & http://www.ummto.dz/ & 13,1 \\
\hline EG & October 6 University & http://www.o6u.edu.eg/ & 13,06 \\
\hline YE & Ibb University & http://www.ibbunv.com.ye/ & 12,94 \\
\hline DZ & $\begin{array}{l}\text { Université Hassiba Benbouali de } \\
\text { Chlef }\end{array}$ & http://www.univ-chlef.dz/ & 12,93 \\
\hline Varios & Arab Open University & http://www.arabou.org/ & 12,9 \\
\hline LB & Haigazian University & http://www.haigazian.edu.lb/ & 12,88 \\
\hline YE & Hodeidah University & http://www.hoduniv.edu.ye/ & 12,87 \\
\hline SA & Girls University In Riyadh & http://www.rug.edu.sa/ & 12,83 \\
\hline LB & Islamic University & http://www.iul.edu.lb/ & 12,69 \\
\hline TN & Université de Gafsa & http://www.ugaf.rnu.tn/ & 12,69 \\
\hline IQ & Salahaddin University & http://www.suh-edu.com/ & 12,55 \\
\hline LB & Beirut University Online & http://www.buonline.edu.lb/ & 12,4 \\
\hline EG & Nahda University & http://www.nahdauniversity.org/ & 12,33 \\
\hline sy & Ittihad Private University & http://www.uu-sy.com/ & 12,31 \\
\hline \multicolumn{4}{|c|}{ ÁREA IV } \\
\hline TN & Time Université & http://www.time.ens.tn/ & 12,31 \\
\hline $\mathrm{AE}$ & Al Ghurair University & http://www.agu.ae/ & 12,14 \\
\hline LY & 7th October University & http://www.7ou.edu.ly/ & 12,02 \\
\hline KW & $\begin{array}{l}\text { American University of The Middle } \\
\text { East }\end{array}$ & http://www.aum.edu.kw/ & 12,01 \\
\hline SD & Ahfad University for Women & http://www.ahfad.org/ & 11,95 \\
\hline YE & Queen Arwa University & http://www.qau.edu.ye/ & 11,94 \\
\hline TN & Université de Tunis & http://www.utunis.rnu.tn/ & 11,91 \\
\hline DJ & Université de Djibouti & http://www.univ.edu.dj/ & 11,88 \\
\hline YE & Aden University & http://www.aden-univ.net/ & 11,87 \\
\hline TN & Université Ezzitouna & http://www.uz.rnu.tn/ & 11,77 \\
\hline SY & Damascus University & http://www.damasuniv.shern.net/ & 11,74 \\
\hline TN & Université de Sfax & http://www.uss.rnu.tn/ & 11,58 \\
\hline $\mathrm{DZ}$ & $\begin{array}{l}\text { Université des Sciences Islamiques } \\
\text { Emir Abdelkader }\end{array}$ & http://www.univ-emir.dz/ & 11,56 \\
\hline TN & University of Kairouan & http://www.univ-k.rnu.tn/ & 11,47 \\
\hline $\mathrm{BH}$ & Bahrain International University & http://www.amaiu.edu.bh/ & 11,45 \\
\hline AE & University of Dubai & http://www.ud.ac.ae/ & 11,33 \\
\hline SA & Taif University & http://www.tu.edu.sa/ & 11,29 \\
\hline MA & Université Ibn Zohr & http://www.univ-ibnzohr.ac.ma/ & 11,24 \\
\hline jo & Jadara University & http://www.jadara.edu.jo/ & 11,2 \\
\hline TN & Université Tunis Carthage & http://www.utc.ens.tn/ & 11,18 \\
\hline sY & $\begin{array}{l}\text { Syrian International University for } \\
\text { Science \& Technology }\end{array}$ & http://www.siust.edu.sy/ & 11,06 \\
\hline TN & $\begin{array}{cccc}\text { Université du } & 7 & \text { Novembre } & \text { à } \\
\text { Carthage } & & & \end{array}$ & http://www.univ7nc.rnu.tn/ & 11,04 \\
\hline LB & Global University & http://www.gu.edu.lb/ & 10,97 \\
\hline $\mathrm{DZ}$ & Université Badji Mokhtar & http://www.univ-annaba.org/ & 10,94 \\
\hline TN & Université de la Manouba & http://www.uma.rnu.tn/ & 10,88 \\
\hline IQ & Baghdad University & http://www.uobaghdad.com/ & 10,74 \\
\hline
\end{tabular}

\begin{tabular}{|c|c|c|c|}
\hline SA & Aljouf University & http://www.ju.edu.sa/ & 10,69 \\
\hline SA & Qassim University & http://www.qu.edu.sa/ & 10,58 \\
\hline SD & Dalanj University & $\begin{array}{l}\text { http://www.dalanjuniversity.edu.s } \\
\mathrm{d} /\end{array}$ & 10,52 \\
\hline IQ & Kerbala University & http://www.uokerbala.edu.iq/ & 10,46 \\
\hline LB & Matn University & http://www.matnu.edu.lb/ & 10,41 \\
\hline EG & Minia University & http://www.minia.edu.eg/ & 10,36 \\
\hline so & Amoud University & http://amouduniversity.org/ & 10,31 \\
\hline so & Burao University & http://www.buraouniversity.com/ & 10,3 \\
\hline TN & Université de Jendouba & http://www.uj.rnu.tn/ & 10,16 \\
\hline LB & Université Libanaisee & http://www.ul.edu.lb/ & 10,11 \\
\hline SA & University of Ha'il & http://www.uoh.edu.sa/ & 10,05 \\
\hline TN & University of Gabes & http://www.univgb.rnu.tn/ & 10,04 \\
\hline SD & $\begin{array}{l}\text { University of the Holly Quran and } \\
\text { Islamic Sciences }\end{array}$ & http://www.quran-unv.edu.sd/ & 9,83 \\
\hline EG & Misr International University & http://www.miuegypt.edu.eg/ & 9,78 \\
\hline so & University of Hargeisa & http://www.hargeisauniversity.ne & 9,73 \\
\hline sY & $\begin{array}{l}\text { Ma'moun Private University for } \\
\text { Science and Technology }\end{array}$ & http://www.must.edu.sy/ & 9,36 \\
\hline TN & $\begin{array}{l}\text { Université Aeronautique et des } \\
\text { Technologies }\end{array}$ & http://www.unat.ens.tn/ & 9,14 \\
\hline YE & Sana'a University & http://www.suye.ac/ & 9,03 \\
\hline JO & AL-Ahliyya Amman University & http://www.amman.edu/ & 8,67 \\
\hline SA & Knowledge International University & http://www.kiu.org/ & 8,58 \\
\hline LB & Middle East University & http://www.meu.edu.lb/ & 8,45 \\
\hline so & Nugaal University & http://www.nugaaluniversity.com & 8,38 \\
\hline SD & University El Mahdi & http://www.elmahdi.edu.sd/ & 8,2 \\
\hline SD & Al-Neelain University & http://www.neelain.edu.sd/ & 8,01 \\
\hline IQ & University of Sulaimani & http://www.univsul.org/ & 7,95 \\
\hline MR & Université Virtuelle Africaine & http://www.uva.mr/ & 7,68 \\
\hline EG & $\begin{array}{l}\text { Modern University for Technology } \\
\text { and Information }\end{array}$ & http://www.mti.edu.eg/ & 7,58 \\
\hline JO & $\begin{array}{l}\text { Jordan University of Science and } \\
\text { Technology }\end{array}$ & http://www.just.edu.jo/ & 7,33 \\
\hline EG & Alexandria University & http://www.alex.edu.eg/ & 7,09 \\
\hline MA & Université Quaraouiyine & $\begin{array}{l}\text { http://www.enssup.gov.ma/etabli } \\
\text { ssements/univquarFes.htm }\end{array}$ & 6,51 \\
\hline SD & Omdurman Islamic University & http://www.oiu.edu.sd/ & 6,44 \\
\hline EG & German University in Cairo & http://www.guc.edu.eg/ & 4,93 \\
\hline TN & Université de Tunis El Manar & http://www.utm.rnu.tn/ & 3,87 \\
\hline SA & Northern Borders University & http://www.nbu.edu.sa/ & 3,8 \\
\hline $\mathrm{AE}$ & British University in Dubai & http://www.buid.ac.ae/ & 2,52 \\
\hline TN & Université de Technologie Privée & http://www.utech-tn.net/ & 2,26 \\
\hline MA & Université Moulay Ismaïl & http://www.umi.ac.ma/ & 2,06 \\
\hline YE & $\begin{array}{l}\text { Hadramout University of Science } \\
\text { and Technology }\end{array}$ & http://www.hust.edu.ye/ & 1,6 \\
\hline TN & Université Internationale de Tunis & http://www.uit.ens.tn/ & 1,1 \\
\hline $\mathrm{BH}$ & Ahlia University & http://www.ahlia.edu.bh/ & 0,7 \\
\hline LB & Lebanese International University & http://www.liu.edu.lb/ & 0,45 \\
\hline
\end{tabular}

Faba-Pérez, Cristina, Vargas-Quesada, Benjamín, Al-Dwairi, K. M. O., Moya-Anegón, Félix. La presencia de los países árabes en Internet: un análisis a través de sus indicadores tecnológicos y de sus universidades. // Ibersid. 8 (2014) 19-31. ISSN 1888-0967. 
